ESAIM: PROCEEDINGS, August 2009, Vol. 28, p. 13-32

M. Ismail, B. Maury \& J.-F. Gerbeau, Editors

\title{
DYNAMIC NUMERICAL INVESTIGATION OF RANDOM PACKING FOR SPHERICAL AND NONCONVEX PARTICLES
}

\author{
Sylvain Faure ${ }^{1}$, Aline Lefebvre-Lepot ${ }^{2}$ and Benoît Semin $^{3}$
}

\begin{abstract}
We simulate the sedimentation in a parallelepipedic container of spheres and nonconvex particles constituted by two overlapping spheres. We use the self-written code SCoPI. Thanks to an efficient handling of contacts between particles, it allowed us to consider up to 100, 000 spheres and 10,000 nonconvex particles. The packing fraction (in bulk and close to a wall) as well as the mean value and the distribution of contacts of the final packings are reported. The results obtained for the classical case of spherical particles (packing fraction: 63.7\%, mean number of contacts: 6) are in agreement with previous studies and validate the algorithm. The packing fraction for nonconvex particles increases and then decreases with respect to the aspect ratio, which is similar to the ellipsoid (convex) case. The number of contacts is different from the number of neighbours, which is of course never the case for spherical particles (convex particles). The number of contacts is discontinuous when slightly increasing the aspect ratio from the spherical case: it is equal to 6 in the spherical case and to 10 in the nonconvex case. These values correspond to the isocounting values, i.e. the number of contacts is twice the number of degrees of freedom. This contrasts with the ellipsoid case, where it sharply but continuously increases. Concerning the number of neighbours, it continuously increases for small aspect ratio (which is similar to the convex particle case), but decreases for higher aspect ratio.
\end{abstract}

Résumé. Nous simulons la sédimentation dans un récipient parallélépipédique de sphères et de particules non convexes formées de deux sphères se chevauchant. Afin de réaliser ces simulations, de nouvelles fonctionnalités ont été ajoutées au logiciel SCoPI initialement développé par A. Lefebvre-Lepot. Grâce à une prise en compte efficace des contacts, il nous a permis de considérer jusqu'à 100,000 sphères et 10,000 particules non convexes. La compacité (en volume et près d'une paroi), ainsi que la valeur moyenne et la distribution du nombre de contacts des configurations finales sont décrites. Les résultats obtenus dans le cas classique de particules sphériques (compacité de 63.7\%, nombre moyen de contacts de 6) sont en accord avec de précédentes études et permettent de valider l'algorithme. La compacité pour les particules non convexes augmente puis diminue en fonction du rapport d'aspect, ce qui est similaire au cas (convexe) des ellipsoïdes. Le nombre de contacts est différent du nombre de voisins, ce qui bien entendu n'est jamais le cas pour des sphères (particules convexes). Le nombre de contacts est discontinu lorsque l'on augmente légèrement le rapport d'aspect à partir d'une sphère: il est égal à 6 dans le cas sphérique et à 10 dans le cas non convexe. Ces valeurs correspondent aux valeurs isostatiques, c'est à dire que le nombre de contacts est égal au double du nombre de degrés de liberté. Ce résultat diffère du cas des ellipsoïdes où il augmente fortement mais continûment. Quant au nombre de voisins, il augmente continûment pour de petits rapports d'aspects (ce qui est semblable au cas des particules convexes), mais décroît pour de plus grands rapports d'aspects.

\footnotetext{
1 CNRS et Université Paris-Sud, Laboratoire de Mathématiques, Bât. 425, 91405 Orsay Cedex, France

2 CNRS, CMAP, Ecole Polytechnique, 91128 PALAISEAU Cedex, France

3 Université Pierre et Marie Curie-Paris 6, Univ Paris-Sud, CNRS, Laboratoire FAST, Bât. 502, 91405 Orsay Cedex, France.
}

(C) EDP Sciences, SMAI 2009 


\section{INTRODUCTION}

Characterization of random granular packings is of primary interest on both practical and fundamental points of view [3]. For example, one can think of short-range atomic order, arrangement of colloidal particles in dense deposits, strength of concrete, granular processing, packing of rice, cellulose fibers in paper. The topic of random packing has been extensively studied, mainly focussing on spherical particles. Indeed, it was seen as a first step to more complex shapes and lot of applications concerned spherical-like particles. However, from the former examples, it is clear that the study of nonspherical particles (anisotropic shapes, nonconvex shapes) is also of great interest and it now becomes prominent. The basic problem is to predict the macroscopic behaviour of these systems from their local structure. For example, global and local packing fraction (density), as well as the topological structure resulting from the network of contacts between grains are of great interest to better understand these materials. In order to carry out such studies, it is necessary to find ways to explore the local configurations that can be reached by random packing and to develop methods to analyze the results.

Experimental study of random granular packings has been the subject of a great amount of researches. A lot of them focuses on the packing of (almost) monosized spheres. For example, experimental results concerning the packing fraction of systems made of monosized spheres can be found in [20,27], the study of contacts for such systems has been addressed in $[2,4]$ and experimental results concerning polydisperse systems can be found in $[11,17]$. The packing fraction of randomly packed monosized spheres is known to be in the range $[55 \%, 64 \%]$, depending on the history of the packing - how the spheres have been poured, has the packing been shaken or not - [12]. The lower value, corresponding to the so-called random loose packing, is rather ill-defined and depends on the friction between spheres [12,27]. The essence of the upper limit (random close packing or maximally random jammed) is still under discussion [30,31]. In [5], a theoretical analysis predicted close random packing to occur for packing fractions equal to $0.64 \pm 0.02$. Experimentally, this limit is obtained by shaking the container and is quite well-defined, but doesn't exceed $63.66 \%$ [27]. It is found to be almost independent of the friction between the spheres for small friction coefficients. However, for larger coefficients, as expected, the packing fraction depends on the friction coefficient: increasing it, the system goes from dense to loose packing (see $[11,17]$ ). Studying the local packing fraction, it has also been shown in [20] that the effects of a wall in such experiments is to induce layering, resulting in a damped oscillation of the local packing fraction from the wall. Regarding contacts, for frictionless spheres, the so-called isocounting conjecture predicts that the total number of degrees of freedom of the system ( 3 translational degrees of freedom for each frictionless spheres) is the total number of contacts, so that the averaged number of contacts (coordination number) per sphere is $6[25,30]$. Experimental methods to estimate the number of contacts have large incertitude [2]. In [4], the mean number of "close contacts" per particle is found to be 6.4 for a configuration of monosized spheres with packing fraction equal to $62 \%$. In [2], packing fraction from $58 \%$ to $64 \%$ are investigated and for each configuration, an average coordination number is computed by using three different methods. It is found to increase with packing fraction, going from 5.5 to 7.5 .

As mentioned at the beginning, packings of nonspherical particles are of great practical interest. One of the objective is to compare the behaviour of these systems with the previous ones, made of spheres. Experiments with rigid fibers [24] showed that density depends upon the shape of the particles and more precisely, upon the aspect ratio (defined as the ratio of the length of the fiber and its thickness). In this study, it seemed that the packing fraction for nonspherical particles was lower than the one obtained for spherical ones and was decreasing with respect to the aspect ratio. However, recent numerical results for spherocylinders [32] showed that for "nearly spherical" particles, packing fraction was greater than for spherical ones and this was confirmed by experiments with ellipsoids in $[8,26]$. In these last studies of packing of ellipsoids, it has been shown that, when the aspect ratio $\alpha$ (defined as the ratio of the semi-axis) deviates from 1, the density increases, reaches a maximum and then strongly decreases $[6,8,26]$. For example, in [26], the maximum of packing fraction is reached for $\alpha=1.63$ and is $12 \%$ above the dense random packing packing fraction for spheres. Such a behaviour has also been observed for particles shapes like cylinders or disks [34]. Concerning the average coordination 
number, the isocounting conjecture predicts that it should be equal to 10 for spheroids and 12 for general ellipsoids. Consequently, the coordination number should undergo a jump at $\alpha=1$.

In order to better understand these complex behaviours, numerous numerical algorithms have been developed. Their objective is to compute dense configurations and to study their properties. As expected, most of these algorithms have been developed to compute configurations for spherical particles. They can be divided into two classes we are going to call geometrical algorithms and dynamic algorithms. By geometrical algorithms we mean methods whose objective is to create dense configurations in order to study them, these configurations being obtained without any underlying physical behaviour of the particles. Some of these methods are beginning by generating randomly the centers of the spheres in a cubic lattice with periodic boundaries. It is the case in [14], where each point is the center of an inner and an outer sphere. The real sphere is the inner one and the algorithm iteratively eliminates overlappings while slowly reducing the outer diameter. The algorithm stops when inner and outer diameters coincide. This algorithm generates packings where only two spheres are actually in contact but lot of spheres are nearly touching. Configurations of 1,000 monosized spheres with mean packing fraction close to $64 \%$ are studied. The number of neighbours (couples of spheres whose distance is lower than a fixed parameter $\eta$ ) converges to 6 when the contraction rate and the parameter $\eta$ go to zero. In [16], randomly generated points are also used and the spheres are growing from these points at a fixed rate. The contacts occurring during the evolution of the particles are treated as elastic contacts. A configuration of 8,000 monosized spheres is created with a mean packing fraction equal to $63.7 \%$ and a mean number of contacts equal to 5.83. Sequential algorithms can also be used to create packings: they consist in positioning spheres one after the other. This type of method has been used in [21] to create random loose packings of spheres in a cylindrical container (higher density equal to 59\%). The author recovers in these configurations the wall effects he already experimentally observed in [20]. The second class of methods, dynamic algorithms, consists in imitating experiments by simulating spheres submitted to forces and undergoing contacts. For example, in $[17,29]$, spheres are falling due to gravity in a cube with periodic boundary conditions and in [1] vibrations of the bottom are added. In [29], the authors use Molecular Dynamics to simulate 20,000 rigid spheres with partially elastic contacts while in [17] the 10,000 spheres are supposed to be deformable. In both cases, the effect of friction on density is recovered and the dense packing situation is obtained for frictionless spheres. In [29], the authors recover isostatic packings for frictionless spheres. A method allowing to simulate the behaviour of spheres in a container is proposed in [28]: a few hundreds of particles, submitted to partially inelastic collisions, are falling in a cylinder. In that case, only loose packing configurations can be reached and the effect of both friction and elasticity are studied. The authors show that, as expected, the density decreases when the friction coefficient increases. To the contrary, the higher is the stiffness, the higher is the density.

Numerical simulations were also developed to simulate packing of nonspherical particles. It essentially consists in geometrical algorithms. Packings of ellipsoids is simulated in [8]: small particles are randomly generated in a cell with periodic boundary conditions and dense packing is made by expanding the particles and using elastic contacts. The studies of the behaviour of packing fraction with respect to the aspect ratio are in agreement with experimental results. Concerning the mean coordination number, it is shown that it sharply but continuously goes from 6 for $\alpha=1$ to a constant value (slightly smaller than 10 or 12) for high values of $\alpha$, which contradicts the isocounting conjecture (see $[6,9,25]$ for theoretical studies of this property). In [32, 33], the following algorithm is used: particles are randomly generated in a cell with periodic boundary conditions, at each step the size of the cell is decreased and the positions of the particles are uniformly dilated, if overlaps occur they are removed, the algorithm stops when it is not possible to remove the overlaps anymore. This method was used to simulate packings of spheroids, spherocylinders and cut spheres. Results for the packing fraction of ellipsoids as a function of the aspect ratio comply with experiments. Moreover, it is shown that the behaviour of cut spheres and spherocylinders of aspect ratio close of 1 is similar to the one of spheroids. For large aspect ratios, cut spheres tend to form nonrandom packing (columnar phase). Numerical simulations of the packing of particles of arbitrary shape (even nonconvex) have also been performed. The geometry of the particles can be described by their volume, given by a collection of spheres [23] or pixels [13]. They can also be defined by their surface, given by a single valued function $\rho(\theta, \varphi)$ in a spherical polar coordinate system like in [7] or discretized 
using a triangulation like in [22]. In all these cases, the packing algorithm is of geometrical type. Their results are often validated by testing the algorithm for spheres or ellipsoids.

In this article we present a dynamic algorithm to simulate the packing of a large collection of particles in a box. We consider two types of particles: spheres and nonconvex particles made of two overlapping spheres. The idea is to be as close as possible to experiments in order to recover most of the physical properties that can be observed experimentally. We focus in this article on constructing dense packings of these particles and study their geometrical properties. However, note that, using a dynamic algorithm also makes it possible to study the dynamical properties of the configurations obtained.

We propose a code allowing to simulate the dynamic of the particles falling in a box, undergoing inelastic collisions and submitted to a random force. The box is chosen to be parallelepipedic but could easily be made cylindrical. The model and the numerical algorithm are described in the first Section. In order to be able to simulate large numbers of particules we use the numerical scheme proposed in [18] for inelastic collisions which is based on a global handling of the contacts. In the second Section, we validate the code with monosized spheres: the influence of the different parameters is studied in order to check the robustness of the code and packing fraction in bulk and close to the walls are studied as well as the mean coordination number. Then, in the last Section, the packing fraction and coordination number for the nonconvex particles is investigated.

\section{Numerical MODELING}

\subsection{Settling of the problem}

\subsubsection{Modeling}

We consider a three dimensional system of $N$ spheres. We denote by $\mathbf{x}_{i} \in \mathbb{R}^{3}$ the position of the center of the $i$ th sphere. Its mass, radius and diameter are denoted by $m_{i}, r_{i}$ and $d_{i}$ respectively.

The spheres are falling in a box under the influence of gravity. A random external force is acting on them and they are supposed to undergo inelastic collisions without solid friction. $\mathbf{f}_{i}$ stands for the external forces exerted on sphere number $i$. It can be made of the force of gravity $\mathbf{f}_{i}^{g}=-m_{i} \mathbf{g}, \mathbf{g}$ being the gravitational acceleration, a random force $\mathbf{f}_{i}^{\text {rand }}$, and a dissipative force $\mathbf{f}_{i}^{\text {dissip }}=-\alpha \dot{\mathbf{x}}_{i}$ with $\alpha>0$.

We define $\mathbf{e}_{i j}=\left(\mathbf{x}_{j}-\mathbf{x}_{i}\right) /\left\|\mathbf{x}_{j}-\mathbf{x}_{i}\right\|$ and we denote by $D_{i j}(\mathbf{x})$ the signed distance between spheres $i$ and $j$ : $D_{i j}(\mathbf{x})=\left\|\mathbf{x}_{j}-\mathbf{x}_{i}\right\|-\left(r_{i}+r_{j}\right)$ (See Fig.1). This distance has to remain positive in order to avoid overlappings and consequently, the set of admissible configurations is

$$
\mathcal{Q}=\left\{\mathbf{x} \in \mathbb{R}^{3 N} \text { s.t. } D_{i j}(\mathbf{x}) \geq 0, \forall i<j\right\}
$$

The particles are supposed to undergo inelastic collisions without solid friction: when they collide, the kinetic energy due to their relative normal velocities is instantaneously dissipated. In the following of this Section, we

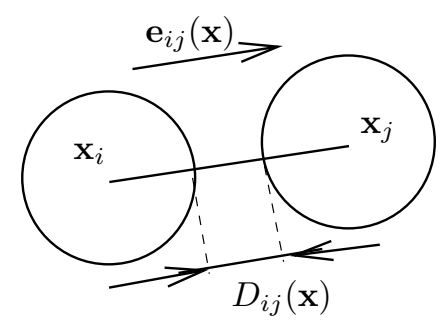

Figure 1. Notations: particles $i$ and $j$.

describe the evolution equations proposed in [18] to model these collisions in case of spherical particles. Then, we explain how it can be used to simulate some kind of nonconvex particles. 


\subsubsection{Evolution equations in case of spherical particles}

Let $\mathbf{x} \in \mathbb{R}^{3 N}$ be defined by $\mathbf{x}=\left(\ldots, \mathbf{x}_{i}, \ldots\right)$ and $\mathbf{f} \in \mathbb{R}^{3 N}$ by $\mathbf{f}=\left(\ldots, \mathbf{f}_{i}, \ldots\right)$. We define $M$ as the mass matrix of dimension $3 N \times 3 N, M=\operatorname{diag}\left(\ldots, m_{i-1}, m_{i-1}, m_{i-1}, m_{i}, m_{i}, m_{i}, m_{i+1}, m_{i+1}, m_{i+1} \ldots\right)$.

We introduce the set of admissible velocities for configuration $\mathbf{x} \in \mathcal{Q}$, which allows to avoid overlappings between particles:

$$
\mathcal{C}_{\mathbf{x}}=\left\{\mathbf{V} \in \mathbb{R}^{3 N} \text { s.t. } \mathbf{G}_{i j}(\mathbf{x}) \cdot \mathbf{V} \geq 0 \text { if } D_{i j}(\mathbf{x})=0, \forall i<j\right\}
$$

where vector $\mathbf{G}_{i j}(\mathbf{x}) \in \mathbb{R}^{3 N}$ is the gradient of the distance between particles $i$ and $j$ with respect to the position of the particles: $\mathbf{G}_{i j}(\mathbf{x})=\nabla_{\mathbf{x}} D_{i j}$. We denote by $\boldsymbol{\lambda}=\left(\ldots, \lambda_{i j}, \ldots\right) \in \mathbb{R}^{N(N-1) / 2}$ the vector made of the Lagrange multipliers associated to these $N(N-1) / 2$ constraints.

Remark 1.1 (obstacles). In our problem, the box consists in $N_{0}=6$ walls which can be considered as obstacles for the particles. In order to take them into account, it is straightforward to add the $N N_{0}$ new constraints of non-overlapping in $\mathcal{C}_{\mathbf{x}}$. For the sake of simplicity, in the following, only particle/particle contacts are considered.

Let $I=] 0, T[$ be the time interval. To write the equations governing the system, we need to define several functional spaces. $\mathrm{W}^{1, \infty}(I)$ is the Sobolev space of functions in $\mathrm{L}^{\infty}(I)$ whose time derivative is also in $\mathrm{L}^{\infty}(I)$. $\mathrm{BV}(I)$ is the space of functions in $\mathrm{L}^{\infty}(I)$ with bounded variations on $I$. If a function $g$ belongs to $\mathrm{BV}(I)$, its limit from the right (resp. from the left) is well-defined for any $t \in I$ and is denoted by $g\left(t^{+}\right)\left(\operatorname{resp} . g\left(t^{-}\right)\right)$. We define the space $\mathcal{M}_{+}(I)$ as the set of positive measures over $I$. To finish with notations, if $K \subset \mathbb{R}^{3 N}, P_{K}$ is the projection operator from $\mathbb{R}^{3 N}$ onto $K$.

The evolution problem presented in [18] is the following:

$$
\begin{gathered}
\mathbf{x} \in\left(W^{1, \infty}(I)\right)^{3 N}, \dot{\mathbf{x}} \in(B V(I))^{3 N}, \quad \boldsymbol{\lambda} \in\left(\mathcal{M}_{+}(I)\right)^{N(N-1) / 2}, \\
\dot{\mathbf{x}}\left(t^{+}\right)=P_{\mathcal{C}_{\mathbf{x}(t)}} \dot{\mathbf{x}}\left(t^{-}\right), \\
M \ddot{\mathbf{x}}=M \mathbf{f}+\sum_{i<j} \lambda_{i j} \mathbf{G}_{i j}(\mathbf{x}), \\
\operatorname{supp}\left(\lambda_{i j}\right) \subset\left\{t, \quad D_{i j}(t)=0\right\} \text { for all } i, j \\
D_{i j} \geq 0 \text { for all } i, j, \\
\mathbf{x}(0)=\mathbf{x}^{0} \text { st. } D_{i j}(0)>0 \text { for all } i, j, \quad \dot{\mathbf{x}}(0)=\mathbf{u}^{0} .
\end{gathered}
$$

Equation (2) consists in a collision law, imposing on the velocities of the particles $\dot{\mathbf{x}}\left(t^{+}\right)$to belong to the set of admissible velocities $\mathcal{C}_{\mathbf{x}(t)}$ for each time $t$. From this minimization problem, for each pair $(i, j)$ with $i<j$, arises a Lagrange multiplier $\lambda_{i j}$ associated to the constraint between particles $i$ and $j$. Equation (3) gives that $\lambda_{i j} \mathbf{G}_{i j}(\mathbf{x})$ can be seen as an additional force, exerted by the particles on each other, due to collisions. Equation (4) says that these forces are active only if the associated particles collide. A straightforward computation gives

$$
\mathbf{G}_{i j}(\mathbf{x})=\nabla_{\mathbf{x}} D_{i j}(\mathbf{x})=\left(\ldots, 0, \quad \underset{i}{-\mathbf{e}_{i j}(\mathbf{x})} \quad, 0, \ldots, 0, \underset{j}{\mathbf{e}_{i j}(\mathbf{x})}, 0, \ldots, 0\right)^{t}
$$

Consequently, the collision between particles $i$ and $j$ creates two forces $\mathbf{f}_{j \rightarrow i}=-\lambda_{i j} \mathbf{e}_{i j}$ and $\mathbf{f}_{i \rightarrow j}=\lambda_{i j} \mathbf{e}_{i j}$, acting on particles $i$ and $j$ respectively. Their modulus are equal to $\lambda_{i j}$ and their sum is equal to zero. They are directed in order to separate the particles involved in the contact (See Figure 2). 


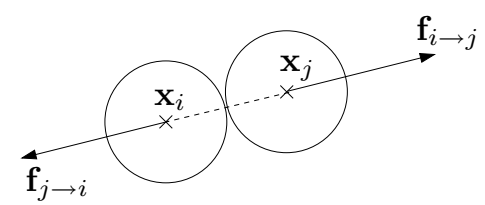

Figure 2. Forces created by a collision between particles $i$ and $j$.

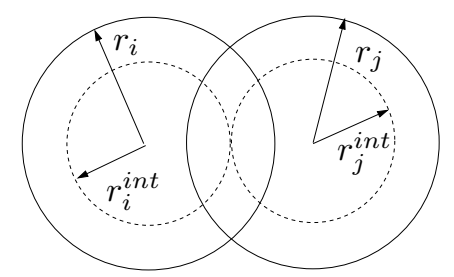

Figure 3. Nonconvex particle made of spheres $i$ and $j$.

\subsubsection{Example of nonconvex particles}

The previous model can be adapted to simulate nonconvex particles made of a collection of spheres. In this article, we consider particles made of two overlapping spheres. In order to control the length of the overlap, we define for each sphere an internal radius. Let's say that spheres $i$ and $j$ belong to the same particle. We denote by $r_{i}^{i n t}$ and $r_{j}^{i n t}$ their respective internal radii. The associated particle is plot on Figure 3 .

We define

$$
\bar{D}_{i j}=\mid \begin{array}{ll}
\left\|\mathbf{x}_{j}-\mathbf{x}_{i}\right\|-\left(r_{i}^{i n t}+r_{j}^{i n t}\right) & \text { if spheres } i \text { and } j \text { belong to the same particle, } \\
D_{i j}(\mathbf{x})=\left\|\mathbf{x}_{j}-\mathbf{x}_{i}\right\|-\left(r_{i}+r_{j}\right) & \text { otherwise, }
\end{array}
$$

and the set of admissible configurations becomes

$$
\overline{\mathcal{Q}}=\left\{\begin{array}{lll}
\mathbf{x} \in \mathbb{R}^{3 N} \text { s.t. } \forall i<j, & \begin{array}{l}
\bar{D}_{i j}(\mathbf{x})=0 \\
\bar{D}_{i j}(\mathbf{x}) \geq 0
\end{array} \quad \text { if spheres } i \text { and } j \text { belong to the same particle, }
\end{array}\right\}
$$

Note that $\nabla \bar{D}_{i j}=\nabla D_{i j}=\mathbf{G}_{i j}$. Consequently, if $\mathbf{x} \in \overline{\mathcal{Q}}$, we define the set of admissible velocities at point $\mathbf{x}$ as

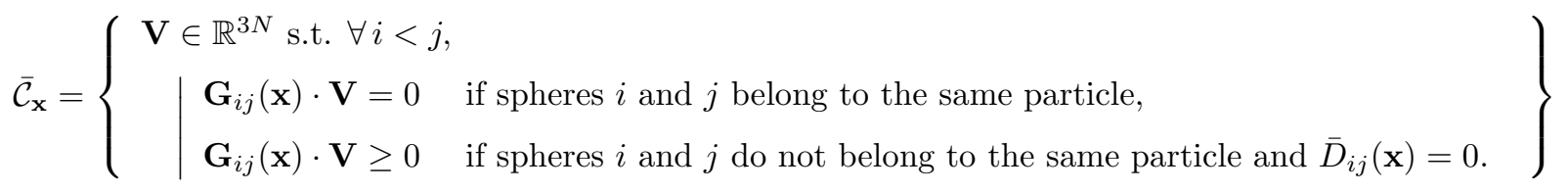

From this, the model (1-6) is straightforwardly extended to this case of nonconvex particles. The random force is exerted independently on each sphere the particle is made up of.

\subsection{Numerical resolution}

\subsubsection{Numerical scheme}

We use the numerical scheme proposed in [18] to solve problem (1-6). The method is based on a global handling of the contacts which allows to make simulations of large numbers of particules. 
Let $h$ be the time step, we denote by $\mathbf{x}^{n} \in \mathbb{R}^{3 N}$ and $\mathbf{V}^{n} \in \mathbb{R}^{3 N}$ the approximated positions and velocities of the spheres at time $t^{n}=n h$. The algorithm is made of two steps. First, we compute an a priori velocity:

$$
\mathbf{V}^{n+1 / 2}=\mathbf{V}^{n}+h \mathbf{f}^{n}
$$

where $\mathbf{f}^{n}=\frac{1}{h} \int_{t^{n}}^{t^{n+1}} \mathbf{f}(s) d s$. This equation consists in an Euler explicit discretization of the Fundamental Principle of Dynamics on each sphere without taking contacts into account. From this, we compute the approximated velocities at time $n+1$ by projecting the a priori velocity onto a set of discrete admissible velocities:

$$
\mathbf{V}^{n+1}=P_{K\left(\mathbf{x}^{n}\right)} \mathbf{V}^{n+1 / 2},
$$

where $K\left(\mathbf{x}^{n}\right)$ is a first order approximation of the constraint $D_{i j}\left(\mathbf{x}^{n+1}\right)=D_{i j}\left(\mathbf{x}^{n}+h \mathbf{V}^{n+1}\right) \geq 0$,

$$
K\left(\mathbf{x}^{n}\right)=\left\{\mathbf{V} \in \mathbb{R}^{3 N} \text { s.t. } D_{i j}\left(\mathbf{x}^{n}\right)+h \mathbf{G}_{i j}\left(\mathbf{x}^{n}\right) \cdot \mathbf{V} \geq 0, \forall i<j\right\} .
$$

Then, the positions of the spheres can be updated:

$$
\mathbf{x}^{n+1}=\mathbf{x}^{n}+h \mathbf{V}^{n+1} .
$$

Remark 1.2 (Projection step and consequences). The projection step of the previous scheme is performed using a Uzawa algorithm. The projection is not exact: a threshold $\varepsilon>0$ is chosen and the projection algorithm is stopped when $D_{i j}\left(\mathbf{x}^{n}\right)+h \mathbf{G}_{i j}\left(\mathbf{x}^{n}\right) \geq-\varepsilon$. As a consequence, as long as two particles are in contact, their distance belongs to $[-\varepsilon, 0]$ and is not necessarily constant. Concerning our simulations, this implies that, even when the random force is off and all the particles are packed at the bottom of the container, a final stationary state may not be reached: the particles still move gently. If necessary, to obtain a still configuration, we will add a dissipation force at the end of the simulation. The influence of parameter $\varepsilon$ will be studied in Section 2.2 .

In order to simulate the nonconvex particles described in Section 1.1.3, this set of discrete admissible velocities has to be modified as follows:

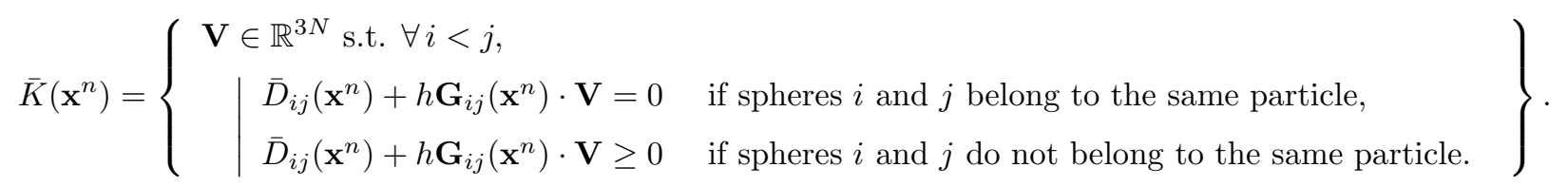

\subsubsection{Programming}

In order to run simulations, we used the code SCoPI initially described in [15]. It is dedicated to the Simulation of Collections of Particles in Interaction. In its original version, the particles are supposed to be spherical and are submitted to external forces such as gravity. It can also take into account moving or still obstacles. The particles can undergo inelastic or gluey collisions and the numerical scheme to deal with these collisions is based on the one described in the previous Section for spherical particles. The object oriented programming method used to build this code makes it possible for the user to add new external forces and collision laws ( [10], [19]).

In order to make it possible to run our simulations using this code, we added a random force to the code, using the Mersenne Twister pseudorandom number generator. We also made it possible to consider spheres with an internal radius and to create particles made of several of these spheres always glued, as described in Section 1.1.3.

Two postprocessing functions were implemented to obtain the parameters characterizing the packing. The first one allows us to compute the packing fraction of the system in a subdomain and is based on a Monte-Carlo method (1,000,000 points are randomly generated in each subdomain). 
Remark 1.3. To validate the function, we considered a cubic box containing a known number of particles of known geometry and not crossing the boundaries of the box (we tested both spherical and nonconvex particles). For more general configurations, where some spherical particules cross the boundaries, the packing fraction values obtained with the Monte-Carlo method were also compared with the exact value : see appendix A for this exact computation.

The second post-processing function gives the distribution and the mean numbers of contacts and of neighbours for the particles contained in a subdomain (box) (the contacts between a particle inside the subdomain and another one outside are taken into account). Notice that in our simulations, we do not need to add an arbitrary criterion to decide whether two spheres are in contact or not: two particles are in contact if the Lagrange multiplier is positive. An important difference between convex and nonconvex particles is that, in the latter case, the number of contacts and the number of neighbours may be different.

\section{VALIDATION: CASE OF MONOSIZED SPHERES}

In this Section, we present our results about the close packing of spheres. The numerical method is validated by comparing our results with those we found in the literature.

\subsection{Protocol}

The spheres are randomly generated in the container of constant size $([0,1] \times[0,5] \times[0,1])$; the size of the particles depends of its number (see Table 2). They are falling due to gravity (the gravity force is along the $y$ direction). At the beginning, the random force is activated. It is stopped and replaced by a dissipation force when the final packing state is approximately reached. In Figure 4, we plot the configurations obtained at four different time steps for the simulation of 100,000 monosized spheres.

\subsection{Parameters affecting the simulations}

The code SCoPI allows one to choose the parameters of the simulation. The effect of the total number of time steps, the random force, the parameter $\varepsilon$ (see remark 1.2) and the number of particles on the obtained packing have been investigated, to check the robustness of the results.

\subsubsection{Number of time steps}

Figure 5 displays the packing fraction and the coordination number as functions of the iteration number, which corresponds to the time in the numerical experiments. The packing fraction and the coordination number are computed in a box strictly smaller than the container $([0.25,0.75] \times[0.25,0.75] \times[0.25,0.75])$, which is completely filled of particles when they have sedimented. When the iteration number increases, the spheres tends to sediment, so that the packing fraction computed in the box at the bottom of the container increases. Its value seems to reach a plateau after about 300 iterations and is constant to within $\pm 0.1 \%$ when the iteration number is greater than 700. The same behaviour is observed for the coordination number (which is constant to within $\pm 0.05 \%$ for iteration number greater than 700$)$. In the following, we focus on the plateau packing.

\subsubsection{Random force}

The effect of the random force is illustrated in Table 1 (lines 2 and 3). The random force allows one to obtain a random packing from an initial regular configuration (which is useful for nonconvex particles). The CPU time needed to reach the plateau values is slightly smaller when adding the random force. The final packing fraction is smaller without random force; yet, the difference is not significant, given that the standard deviation is high and the number of simulations not so large (the number of spheres in the box is small, which may explain, at least partly, the observed fluctuations).

When adding a random force, the obtained coordination number is too small (of about 0.5), because the agitation prevents some contacts from occurring. Therefore, the random force is switched off when the final packing state is approximately reached (and we add a dissipation force which avoid oscillations of the values, 

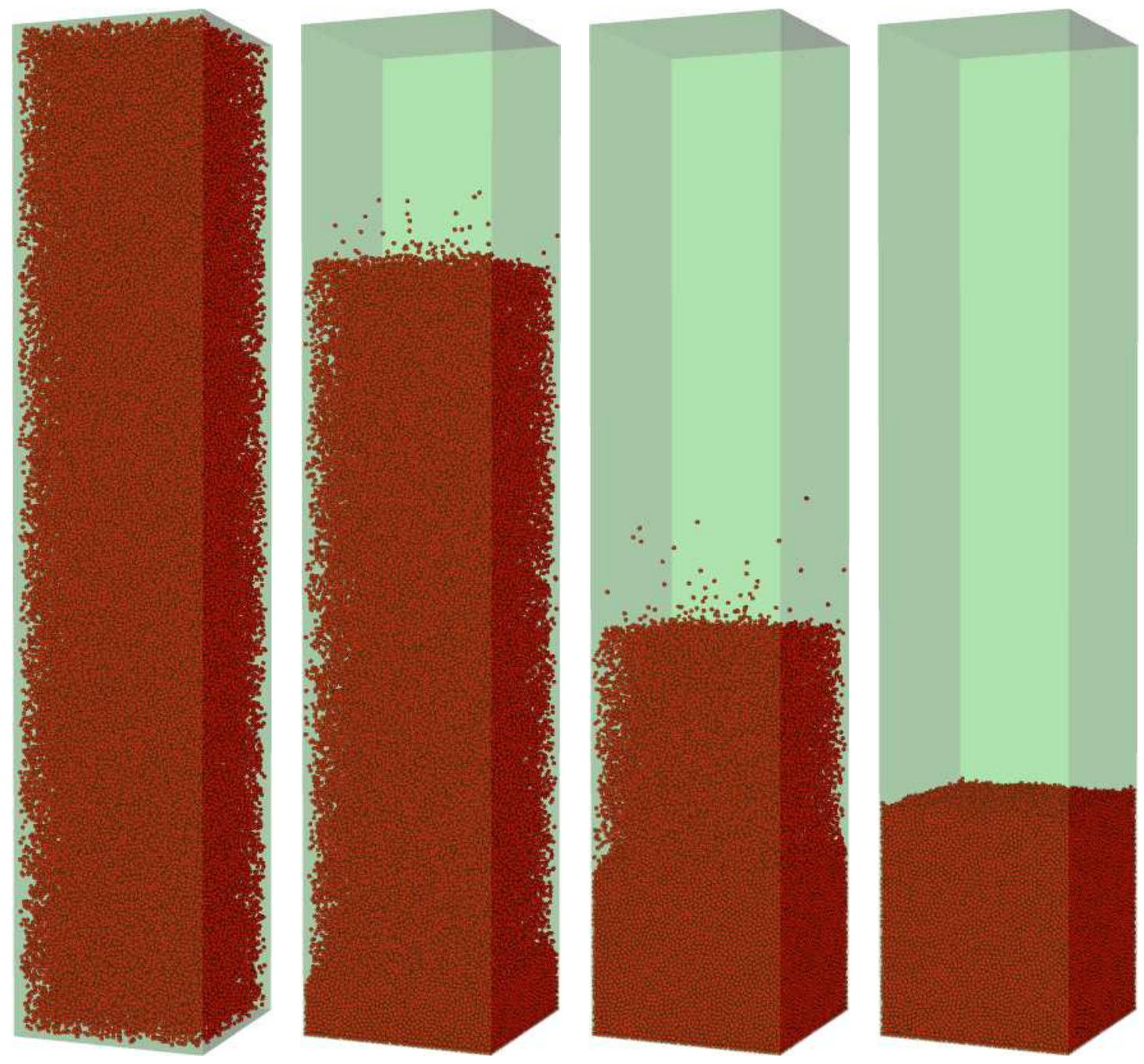

Figure 4. Four configurations for a simulation of 100,000 spheres (initial configuration on the left).

TABLE 1. Effect of $\varepsilon$ and of the random force on the value of the packing fraction and the coordination number for 1,000 spheres $(d=0.11)$. For each protocol, the mean and the standard deviation are obtained from a serie of 9 simulations.

\begin{tabular}{|cc|cc|cc|}
\hline \hline$\varepsilon$ & Random force & \multicolumn{2}{|c|}{ Packing fraction (\%) } & \multicolumn{2}{c|}{ Coordination number } \\
& & mean & standard deviation & mean & standard deviation \\
\hline 0.005 & yes & 63.39 & 0.41 & 6.18 & 0.075 \\
0.0005 & yes & 63.22 & 0.44 & 5.97 & 0.12 \\
0.0005 & no & 63.06 & 0.31 & 5.99 & 0.12 \\
0.00005 & yes & 63.44 & 0.44 & 5.93 & 0.13 \\
\hline \hline
\end{tabular}

see remark 1.2). In the following of the article, the reported values for the simulation are taken after switching off the random force. The coordination number is then the same with and without random force. 

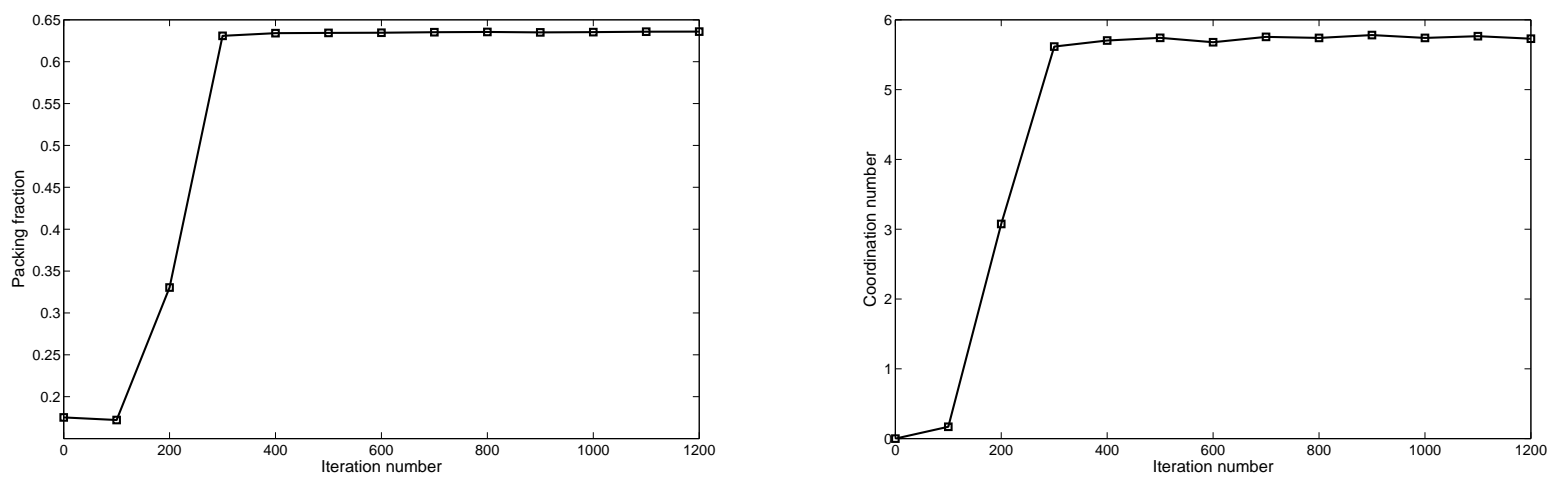

FIGURE 5. Evolution of the packing fraction (left) and coordinence (right) with the iteration number (time) for 10,000 spheres. These quantities are computed in a small box located at the bottom of the container.

\subsubsection{Parameter $\varepsilon$}

The effect of $\varepsilon$, which corresponds to the maximum overlap between different particles (see remark 1.2), is illustrated in Table 1 (lines 1,2,4). When $\varepsilon \leq 0.005 r$, where $r$ is the common radius of the spherical particules, this parameter is seen to have a negligible effect on the packing fraction value. To precisely obtain the coordination number, a smaller value $(\varepsilon \leq 0.0005 r)$ is needed. Since the CPU time increases when $\varepsilon$ is decreased, we have chosen $\varepsilon=0.0005 r$ in the simulations, unless otherwise specified.

\subsubsection{Number of particles}

When the sphere diameter is large (or, more precisely, the sphere diameter to wall distance ratio), wall effects are important and the values in the box are not the bulk ones. Table 2 shows that the results about the packing fraction are quite insensitive to the particle number (linked in our simulations to its diameter) when using more than 10,000 particles.

TABLE 2. Effect of the number of particles on the value of the packing fraction and the coordination number.

\begin{tabular}{|cccc|c|}
\hline \hline Number of particles & $d$ & $\varepsilon$ & Number of simulations & Packing fraction (\%) \\
\hline 1,000 & 0.11 & 0.0005 & 9 & 63.2 \\
10,000 & 0.052 & 0.0005 & 2 & 63.6 \\
100,000 & 0.024 & 0.005 & 1 & 63.7 \\
\hline \hline
\end{tabular}

\subsection{Packing fraction}

We found a packing fraction of $63.7 \pm 0.2 \%$, which is in agreement with previous results for random close packing $[12,16,27]$. This agreement validates our numerical method.

We have also addressed the problem of the wall effects. Walls are indeed known to induce layering [20]. We measure the packing fraction in boxes $[l, l+\delta] \times[0.25,0.75] \times[0.25,0.75]$, where $l \in[0,0.25]$ and $\delta$ is chosen an order of magnitude smaller than the spheres diameter $(\delta=0.005$ for samples of 10,000 spheres of diameter 0.052). Figure 6 displays the local packing fraction as a function of the dimensionless distance from the wall, for simulations where the sphere diameter ranges between 0.11 and 0.024 . The packing fraction has a damped oscillating behaviour, consistent with previous studies [20]. The curves corresponding to several sphere diameters collapse on a master curve, which shows that the wall effects do not depend on the sphere diameter (or, more 


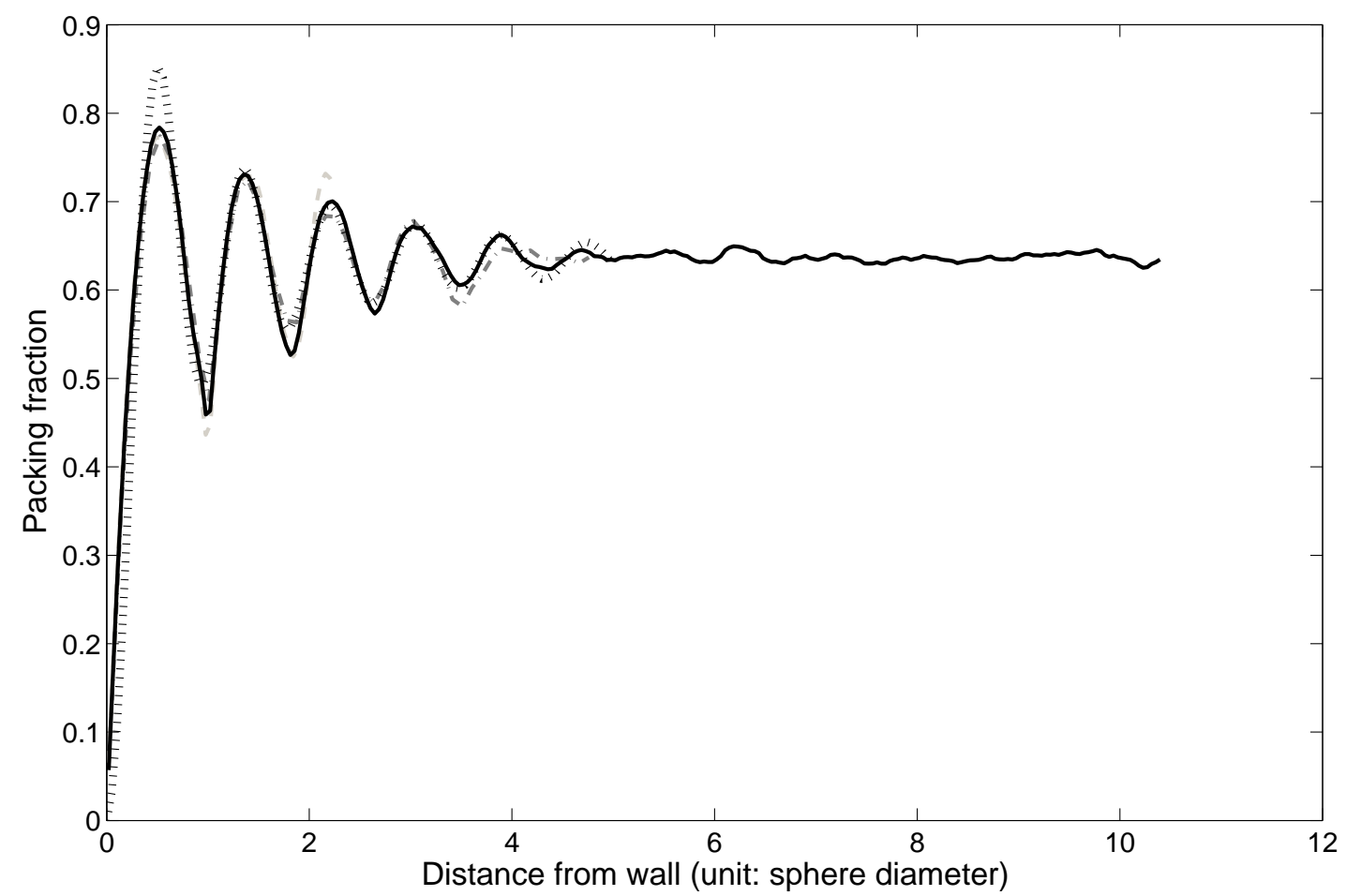

FiguRE 6. Local packing fraction as a function of the distance from the wall. Dashed line : 1,000 spheres, $d=0.11$; dashed dotted line : 10,000 spheres, $d=0.052$; solid line : 100,000 spheres, $d=0.024$; dotted line : empirical model from $[20]$

precisely the ratio of the sphere diameter to the container length). Therefore, the results we have obtained for spherical and probably also for nonconvex particles are likely to correspond to the case of an infinite wall. Mueller [20] studied the wall effects in a cylindrical container, and deduce an empirical formula of the packing fraction as a function of the wall distance. He found that the wall effects depend on the sphere diameter to container diameter ratio. This is not the case in our simulations, which suggests that this dependency is due to the curvature of the container. The empirical formula of Mueller, in the case of an infinitely wide container, is displayed for comparison in Figure 6 (note that using Mueller's formula for the packing fraction in a finite diameter container gives a less good agreement).

\subsection{Coordination number}

Figure 7 displays the distribution of the coordination number (number of contacts, which is equal to the number of neighbours for spherical particles because a sphere is convex), for two different simulations in order to show the reproducibility of this result. The mean coordination number is $6.00 \pm 0.05$, which is in agreement with previous studies $[6,8,25]$. The most frequent coordination number is 6 , the distribution is a little asymmetric with respect to this value. No sphere has a coordination number of 0 or 1 because such configurations are mechanically unstable. We found no sphere with 11 or 12 neighbours, in agreement with [16].

A graph of the network of contacts obtained for 10,000 spheres can be found on Figure 8 . 


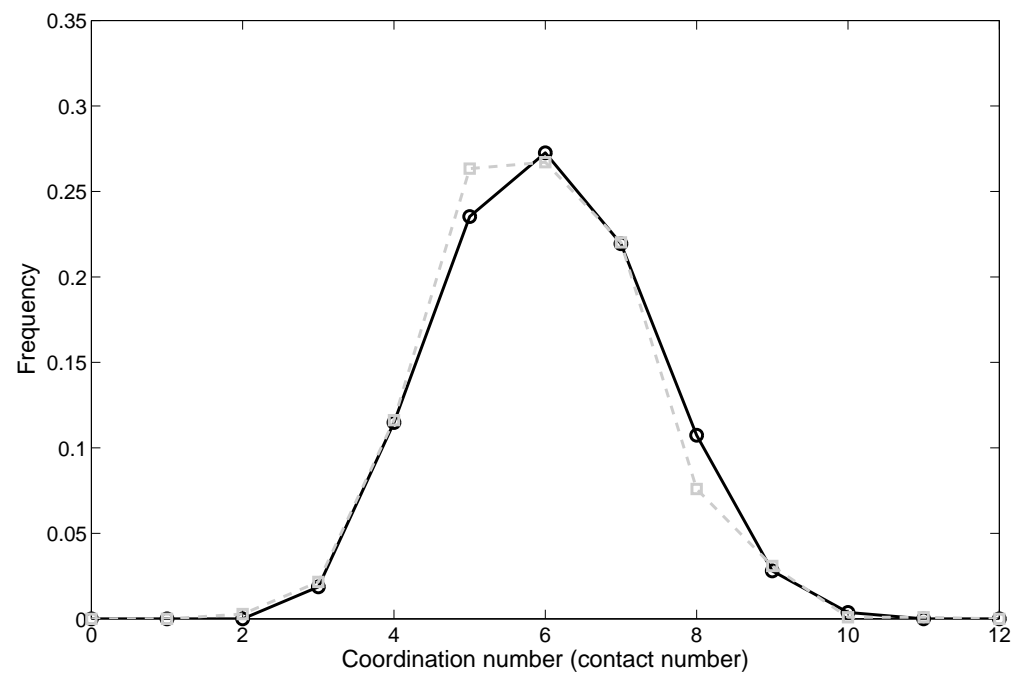

Figure 7. Distribution of the coordination number (number of contacts) for two curves corresponding to two different simulations with the same parameters $(10,000$ spheres, $\varepsilon=0.0005 r$.

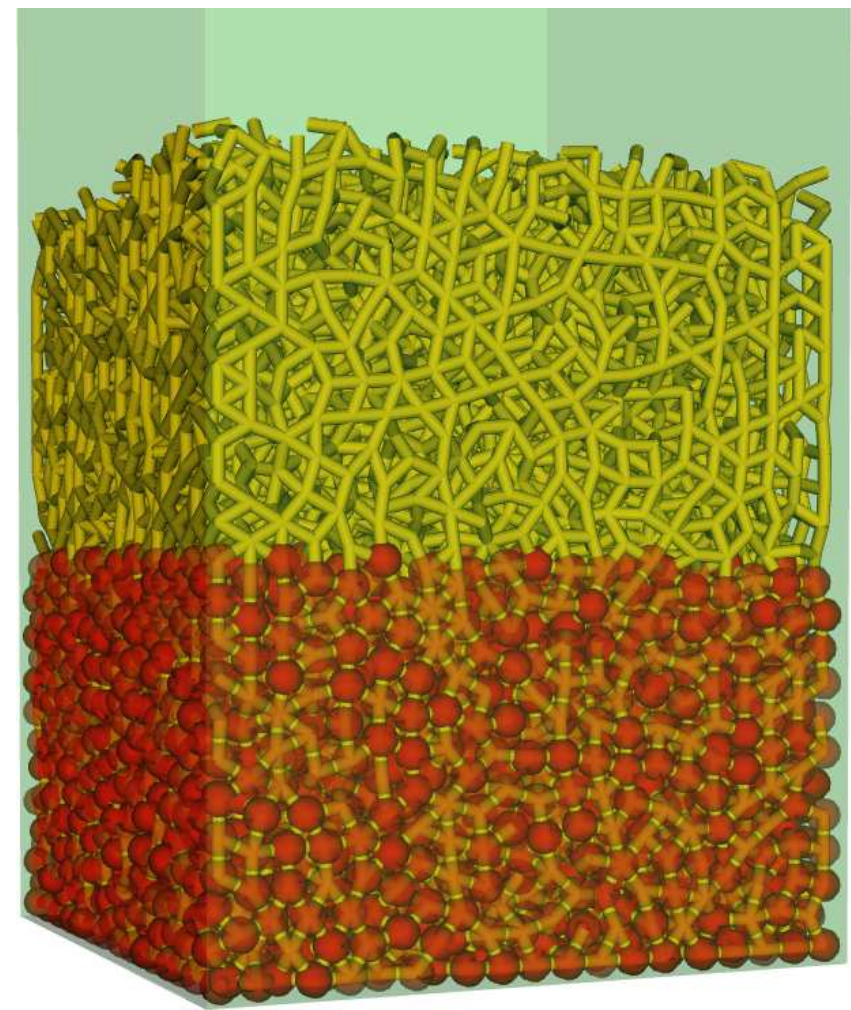

Figure 8. Network of contacts obtained for the packing of 10000 spheres (only the spheres situated at the lower half of the configuration are represented). 


\section{NONCONVEX PARTICLES}

\subsection{Protocol}

We consider the nonconvex particles made of two overlapping spheres, described in Section 1.1.3. The simulation of such a particle is given on Figure 9.

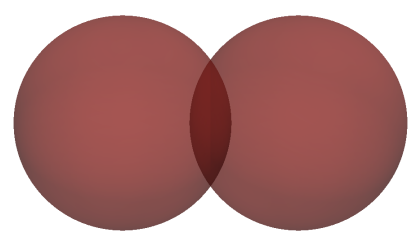

Figure 9. Simulation of a nonconvex particle.

The protocol is similar to the one described for spheres in Section 2.1. We simulate the sedimentation of 10,000 of these particles in the same container. The diameter of the spheres making the particles is $d=0.052$. For simplicity, the initial configuration is not randomly generated. In Figure 10, we plot different configurations obtained at four different time steps.

Since the initial configuration is not random, we have studied the isotropy of the packing. Let $a_{x}^{2}$ be defined as the mean (in an inner box) of $\left(x_{i}-x_{j}\right)^{2}$, where the spheres $i$ and $j$ belong to the same nonspherical particle. Let $R$ be the distance between the centers of the two spheres constituting a nonconvex particles. For an isotropic packing, $a_{x}=a_{y}=a_{z}=R / \sqrt{3}$. We have found that $a_{x} \approx a_{z}$. However, $a_{y}<a_{x}$ and none of these values is equal to the expected one for an isotropic packing (for example, for an aspect ratio of 1.6, $a_{x}=0.0260$, $a_{y}=0.0191, a_{z}=0.0262, R / \sqrt{3}=0.0240$; the anisotropy increases with the aspect ratio). This means that the $x$ and $z$ directions are equivalent (no memory of the initial configuration). The packing is not isotropic because the gravity force induce a particular direction $(y)$ of the packing. This contrasts with packing obtained by geometrical algorithms $[6,8]$, which are isotropic. As for our simulations, experimental packings are expected to be anisotropic.

\subsection{Packing fraction}

We have investigated the variation of the packing fraction with the aspect ratio of the particle, defined as $d / L$, where $d$ is the diameter and $L$ the length of the particle (see Fig. 11). Curve $\square$ of Figure 11 shows that the packing fraction first increases when increasing the aspect ratio and decreases above about 1.5. This behaviour is similar to the one obtained by Donev for ellipsoids (Fig. 1, [9], maximum value of 71\% reached for an aspect ratio of 1.5). This means that the packing fraction is quite insensitive to the precise shape and to the convexity of the particles. Since the particle are nonconvex (for $d / L>1$ ), a volume near the junction of the two spheres composing the particle cannot be reached by the other particles. Adding (by thought) this excluded volume (see Fig. 11) has no effect on the simulation, but increases the packing fraction. The obtained curve ( $\diamond$ of Figure 11 ) is qualitatively similar to the raw one.

Figure 12 displays the packing fraction as a function of the distance from the wall, for different aspect ratio particles. The distances are made dimensionless using the diameter of the spheres which constitute the nonconvex particles. When the aspect ratio is close to 1 , the curve $(\square)$ is quite similar to the spherical case $(\triangle)$, with a greater period. For intermediate aspect ratios particles ( $\circ$ and + ), the first period is quite well-defined and the curve is more irregular (and not very reproducible) for greater distances. When the aspect ratio is close to 2 (two spheres glued without overlapping), the curve is very close to the curve for independent spheres; this latter effect is likely to be due to the specific shape of the particles. 

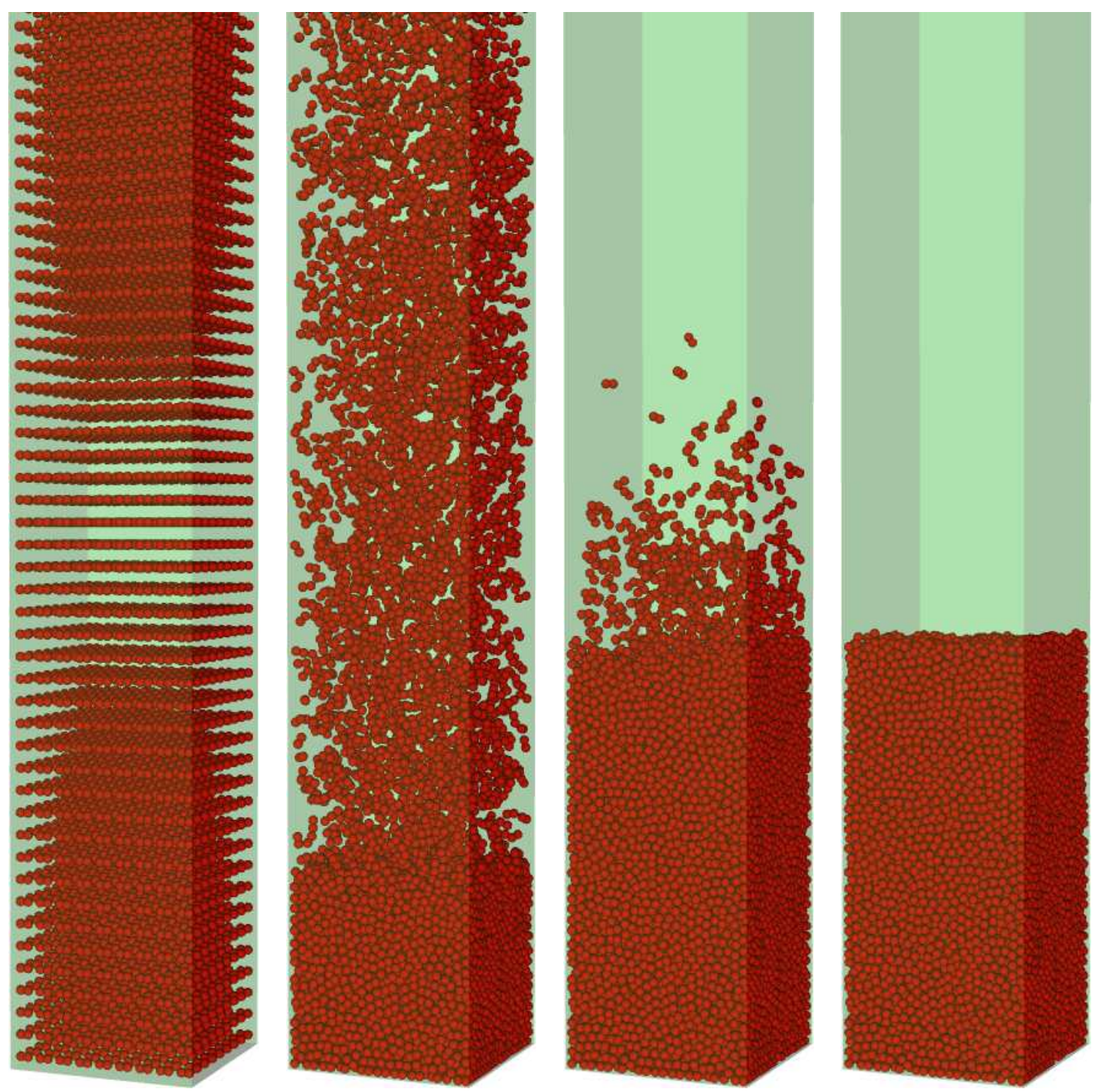

Figure 10. Four configurations for a simulation of 10,000 nonconvex particles (initial configuration on the left).

\subsection{Numbers of contacts and of neighbours}

The effect of the aspect ratio on the number of neighbours and the number of contacts is illustrated in Figure 13. Since the particles are nonconvex, two particles can undergo several contacts one with the other, and consequently, the corresponding curves are different. The number of contacts is constant and equal to $10 \pm 0.2$ when $d / L>1$. This value corresponds to the isostatic value: the number of contacts is twice the number of degrees of freedom of the system (3 degrees of translation and only 2 of rotation, because of the symmetry of the particle). This result contrast with the result obtained for ellipsoids [9], where the number of contacts continuously increases with the aspect ratio. Similarly to the packing fraction, the number of neighbours increases continuously and then decreases. The beginning of the curve is quite similar to the one obtained in [9] for ellipsoids. This may be due to the fact that for nonspherical particles of small aspect ratio, 

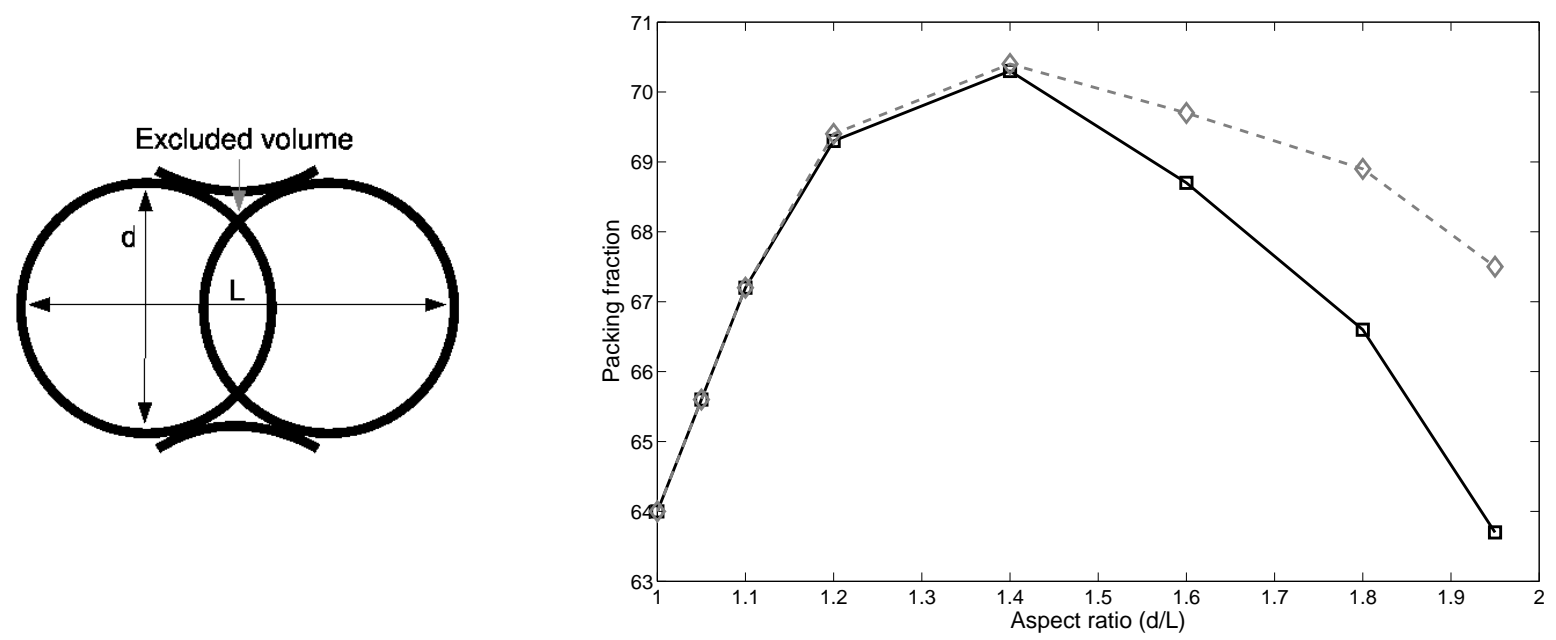

FIGURE 11. Schematic view of a nonconvex particle (left). Packing fraction as a function of the aspect ratio of the particles. $\square$ : raw values; $\diamond$ : packing fraction when adding the excluded volume(right) $(\varepsilon=0.005)$.

the number of neighbours is geometrically constrained to be only slightly different of the one of the spherical case. Contrarily to the nonconvex case, for spherical particles, the number of contacts and neighbours being equal, this should impose a continuous variation of the contact number. The mean number of neighbours is always smaller than 10, which means that there is always multiple contacts between neighbouring particles.

The distributions of the number of contacts and of neighbours are displayed in Figure 14. The distribution of the number of contacts is almost the same for all the aspect ratios (the distribution is slightly narrower for $d / L=0.4$ and $d / L=0.6)$. The number of contacts ranges between 4 and 18 in most of our simulations, and the most probable value is 10 . The number of neighbours ranges between 4 and 12, and the distribution depends on the aspect ratio, which is consistent with the variation of the average number of neighbours with the aspect ratio.

\section{CONCLUSION}

We have characterized packings obtained with a self-written code (SCoPI). This dynamic code allows us to simulate many particles (an example for as much as 100,000 particles is reported) with a very accurate handling of contacts between particles. We have simulated the sedimentation of particles in a parallelepipedic container. The particles are submitted to a random force at the beginning of the simulation, in order to obtain random configurations.

First, we have validated the code with spherical particles, for which experimental and numerical results can be found in the literature. Both the bulk packing fraction (63.7\%) and the bulk coordination number $(6.00 \pm 0.05)$, which is obtained without the need of an arbitrary contact criterion, are in good agreement with previous results. We have also reported the distribution of the contact number. The wall effects we found are in qualitative agreement with the cylinder case, but are found to be independent of the sphere to container ratio: we can so infer we have measured the effect of a single wall.

Then, we have measured the same quantities for nonconvex particles constituted by two overlapping spheres. The packing fraction increases and then decreases with the aspect ratio, in agreement with the case of ellipsoids. The obtained packings are anisotropic (the gravity direction is not equivalent to the others), as expected in "real" packings, which is in contrast with the packing obtained by geometrical algorithms. For small aspect 


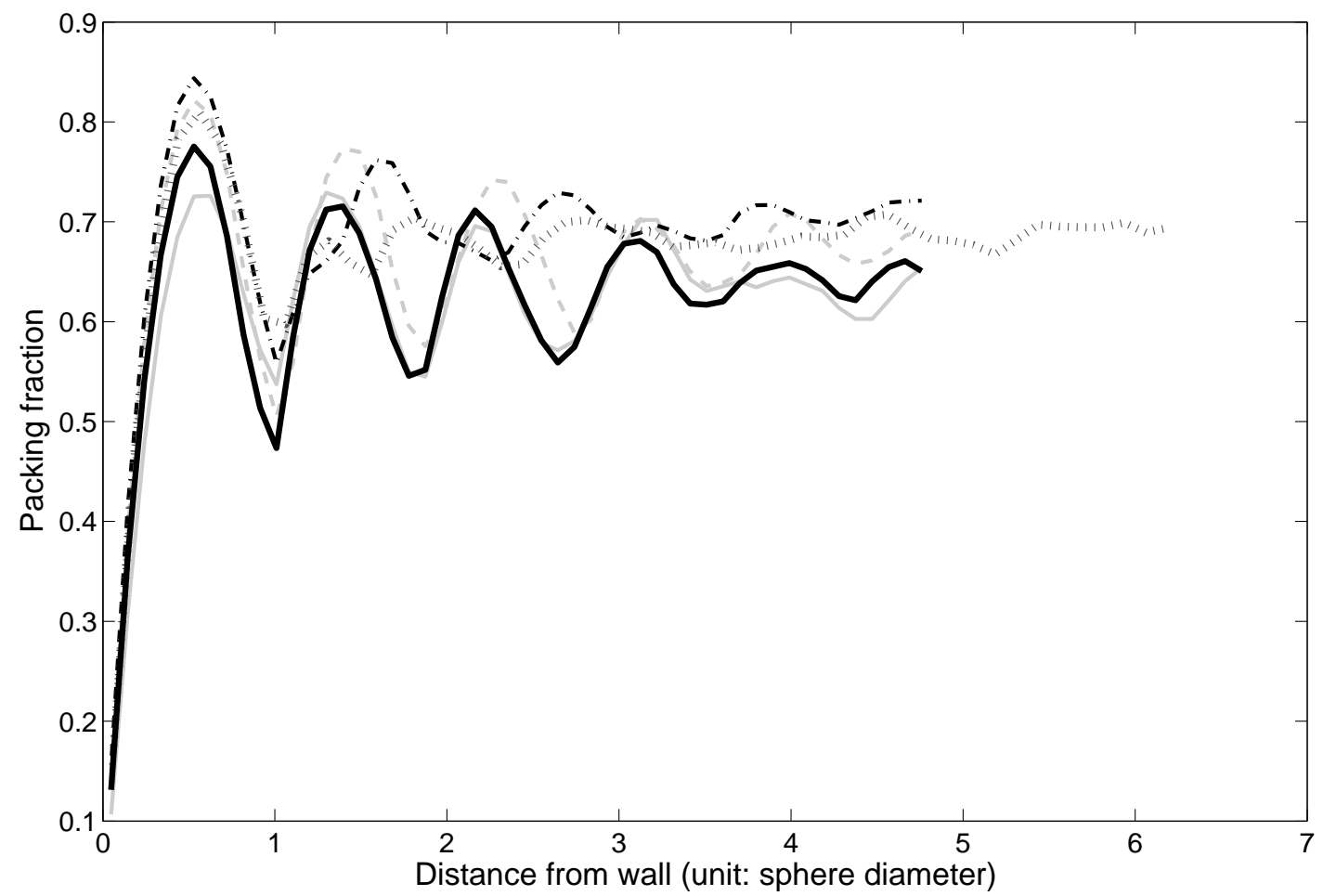

FIGURE 12. Wall effects for particles of different aspect ratios $(\varepsilon=0.005 r)$. Black solid line: spheres $(d / L=1)$; gray dashed line: aspect ratio $d / L=1.1$; black dashed dotted line: $d / L=$ 1.4 ; black dotted line: $d / L=1.6$; gray solid line: $d / L=1.95$

ratio, the wall effects are similar to the sphere case, which is not the case for higher aspect ratio particles (note that, for aspect ratio of about 2, i.e. no overlap between spheres, we recover wall effects quite similar to the sphere case, which may be due to the special choice of the nonconvex particles). For the nonconvex particles, the number of contacts and the number of neighbours are different. The number of contacts seems to discontinuously increases from 6 to 10 when the aspect ratio increases: the isocounting conjecture, which states that the number of contacts is twice the number of degrees of freedom per particle is verified; this is not the case for ellipsoids. For small aspect ratio, the variation of the number of neighbours is similar to the convex case. Yet, this number is always smaller than 10 and decreases for high aspect ratio.

This study opens up new prospects, as example, the study of polydisperse particles will be possible with the code. Moreover, since the algorithm is a dynamic one, it will also be possible to study the response of the packing to a change of the stress or of the configuration (by example by removing the lateral walls).

We gratefully acknowledge Philippe Gondret and Bertrand Maury for stimulating discussions. We thank Lafarge for providing us a financial support.

\section{A. Exact COMputation of the PACKing FRACTiOn}

In order to compute the packing fraction of a configuration in a cube, it is clearly sufficient to be able to compute the volume of the intersection of a sphere and a cube. So, let us consider a sphere of center $I$ and radius 


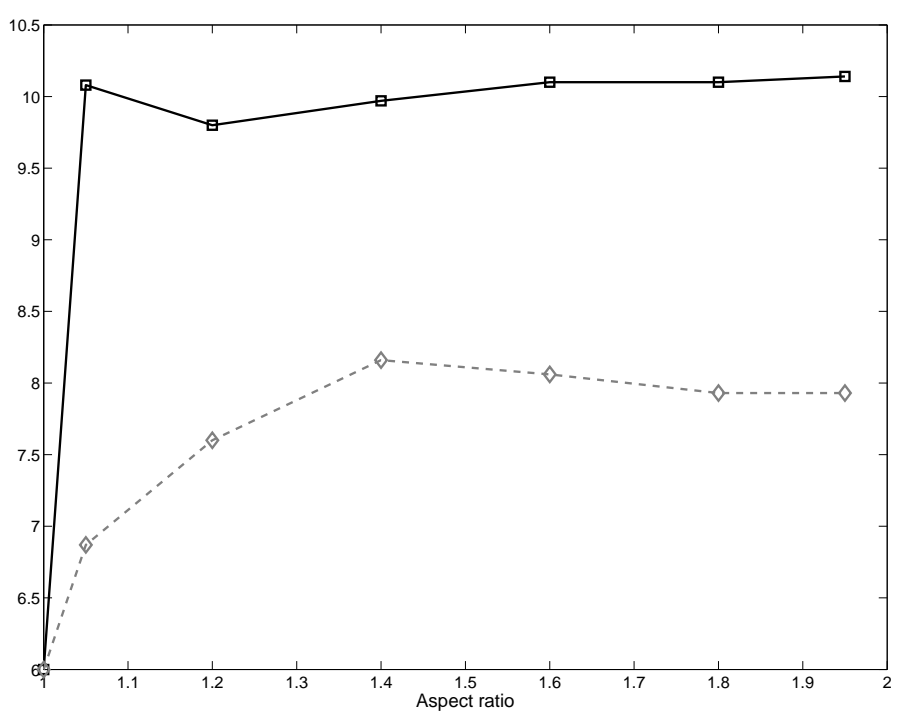

FIGURE 13. Number of neighbours and number of contacts as a function of the aspect ratio of the particles $(\varepsilon=0.0005 r) . \square$ : number of contacts; $\diamond:$ number of neighbours
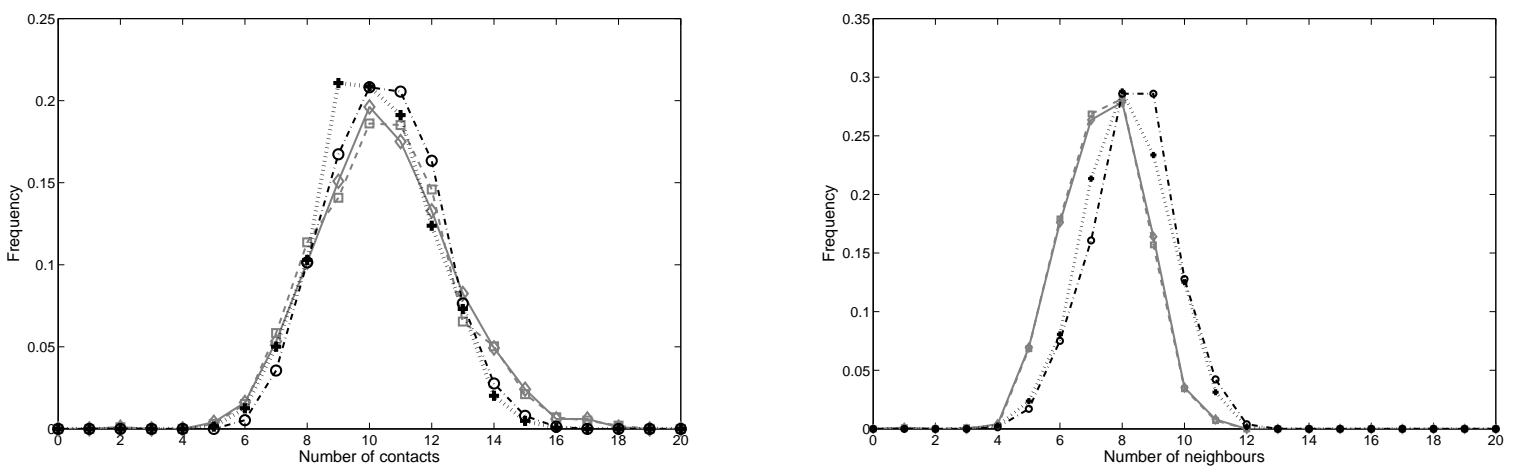

FiguRE 14. Distribution of the number of contacts (left) and neighbours (right) for different aspect ratio particles $(\varepsilon=0.005 r)$. Same line symbols as Figure 12

$R$, and a cube with side greater than $2 R$. To compute the volume of their intersection, we have to consider six cases :

- the sphere is in the cube,

- the sphere crosses one face of the cube,

- the sphere crosses two faces of the cube,

- the sphere crosses two faces and the corresponding edge of the cube,

- the sphere crosses three faces of the cube,

- the sphere crosses three faces and one edge of the cube,

- the sphere crosses three faces and two edges of the cube,

- the sphere crosses three faces and three edges of the cube, 
- the sphere crosses three faces and three edges with the corresponding corner of the cube,

The computation of the corresponding volumes can straightforwardly be achieved by combining three particular volumes. In the following we describe and compute these volumes.

\section{Case of a face :}

In this Section, we suppose that the sphere crosses a face of the cube. An example of this situation, as well as the corresponding notations, are given in Figure 15. The parameter describing this configuration is $a \in[-R, R]$
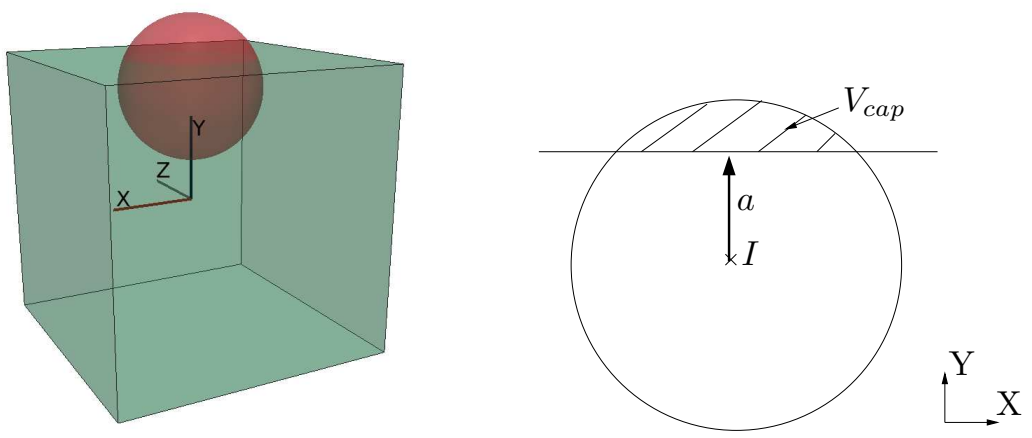

Figure 15. Case 1 : the sphere crosses a face of the cube. Configuration (left) and 2Dprojection to set the notations

(see Figure 15). In that case, the volume of the cap is:

$$
V_{\text {cap }}=\pi\left(\frac{2}{3} R^{3}-a R^{2}+\frac{a^{3}}{3}\right)
$$

\section{Case of an edge :}

Let us now suppose that the sphere crosses an edge of the cube. We compute the volume $V_{\text {edge }}$ described on Figure 16. The parameters are $(a, b) \in[0, R] \times\left[0, r_{m}\right]$, where $r_{m}=\sqrt{R^{2}-a^{2}}$ (see Figure 16). The volume is
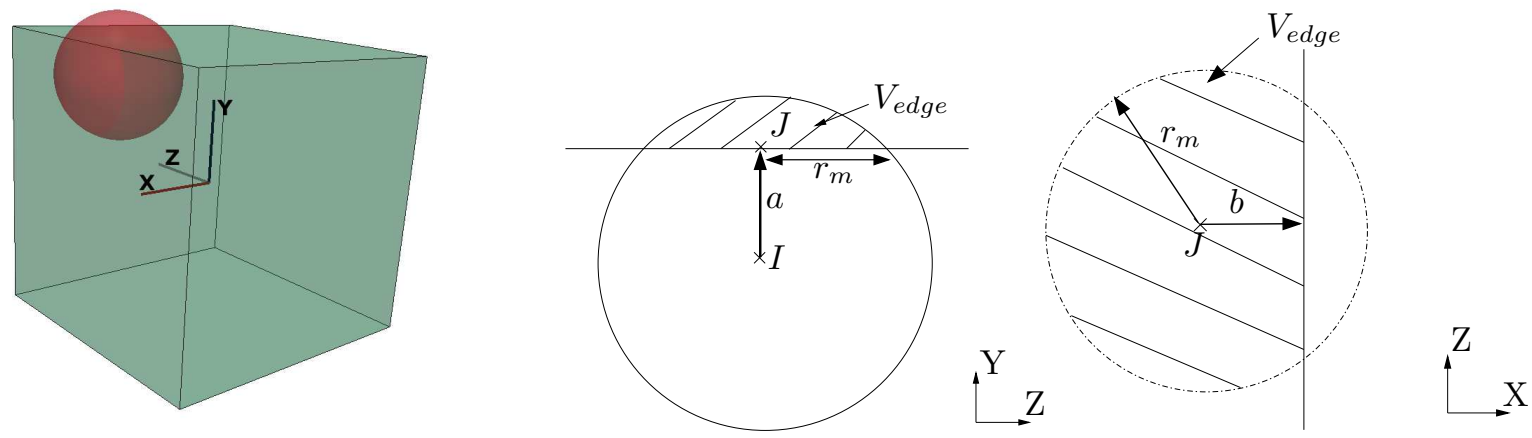

Figure 16. Case 2 : the sphere crosses an edge of the cube. Configuration (left) and 2Dprojection to set the notations

given by

$$
V_{\text {edge }}=\left(-\pi+2 \theta_{0}\right) I\left(r_{m}\right)+\int_{\theta_{0}}^{\pi-\theta_{0}} I\left(\frac{b}{\sin \theta}\right) \mathrm{d} \theta+\frac{2 \pi R^{3}}{3}-a\left(b \sqrt{r_{m}^{2}-b^{2}}+r_{m}^{2}\left(\arcsin \left(\frac{b}{r_{m}}\right)+\frac{\pi}{2}\right)\right),
$$


where $\theta_{0}=\arcsin \left(\frac{b}{r_{m}}\right)$ and $I(r)=-\frac{1}{3}\left(R^{2}-r^{2}\right)^{3 / 2}$.

\section{Case of a corner :}

The last volume we need to compute is described in Figure 17.
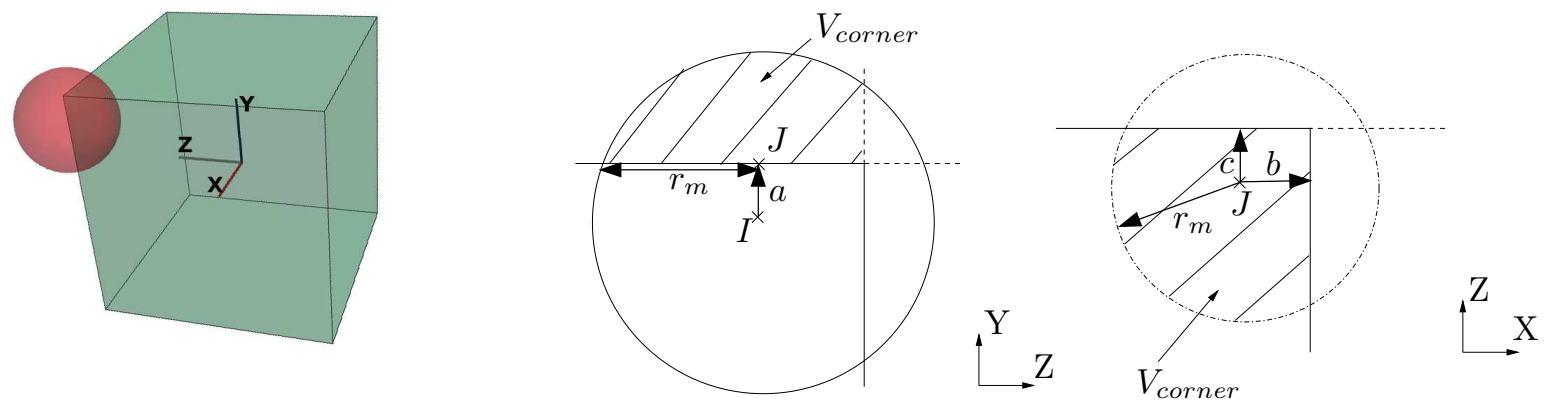

Figure 17. Case 3 : the sphere crosses a corner of the cube. Configuration (left) and 2Dprojection to set the notations

The parameters are $(a, b, c) \in[0, R] \times\left[0, r_{m}\right] \times\left[0, r_{m}\right]$ where $r_{m}=\sqrt{R^{2}-a^{2}}$ (see Figure 17) and the volume is equal to

$$
\begin{aligned}
V_{\text {corner }}= & \left(\pi+\theta_{0}-\theta_{1}\right) I\left(r_{m}\right)+\int_{-\theta_{1}}^{\theta_{2}} I\left(\frac{c}{\cos \theta}\right) \mathrm{d} \theta+\int_{\theta_{2}}^{\pi-\theta_{0}} I\left(\frac{b}{\sin \theta}\right) \mathrm{d} \theta+\frac{2 \pi R^{3}}{3} \\
& -a\left\{\frac{1}{2}\left[b \sqrt{r_{m}^{2}-b^{2}}+r_{m}^{2} \arcsin \left(\frac{b}{r_{m}}\right)-y_{c} \sqrt{r_{m}^{2}-y_{c}^{2}}+r_{m}^{2} \arcsin \left(\frac{-y_{c}}{r_{m}}\right)\right]+\frac{\pi}{2} r_{m}^{2}+c\left(b+y_{c}\right)\right\},
\end{aligned}
$$

where $\theta_{0}=\arcsin \left(\frac{b}{r_{m}}\right), \theta_{1}=\arccos \left(\frac{c}{r_{m}}\right), \theta_{2}=\arctan \left(\frac{b}{c}\right), I(r)=-\frac{1}{3}\left(R^{2}-r^{2}\right)^{3 / 2}$ and $y_{c}=\sqrt{r_{m}^{2}-c^{2}}$. 


\section{REFERENCES}

[1] X.Z. An, R.Y. Yang, R.P. Zou, and A.B. Yu. Effect of vibration condition and inter-particle frictions on the packing of uniform spheres. Powder Technology, 188:102-109, 2008.

[2] T. Aste, M. Saadaftar, and T. J. Senden. Local and global relations between the number of contacts and density in monodisperse sphere packs. Journal of Statistical Machanics: Theory and Experiment, page P07010, 2006.

[3] T. Aste and D. Weaire. The Pursuit of Perfect Packing. CRC Press, 2000.

[4] J. D. Bernal and J. Mason. Co-ordination of randomly packed spheres. Nature, 188:910-911, 1960.

[5] J. G. Berryman. Random close packing of hard spheres and disks. Phys. Rev. A, 27(2):1053-1061, 1983.

[6] P. M. Chaikin, A. Donev, W. Man, F. H. Stillinger, and S. Torquato. Some observations on the random packing of hard ellipsoids. Ind. Eng. Chem. Res., 45:6960-6965, 2006.

[7] D. Coelho, J.-F. Thovert, and P. M. Adler. Geometrical and transport properties of random packings of spheres and aspherical particles. Phys. Rev. E, 55:1959-1978, 1997.

[8] A. Donev, I. Cisse, D. Sachs, E. A. Variano, F. H. Stillinger, R. Connelly, S. Torquato, and P. M. Chaikin. Improving the density of jammed disordered packings using ellipsoids. Science, 303:990-993, 2004.

[9] A. Donev, R. Connelly, F. H. Stillinger, and S. Torquato. Underconstrained jammed packings of nonspherical hard particles: Ellipses and ellipsoids. Phys. Rev. E, 75:051304, 2007.

[10] S. Faure, S. Martin, B. Maury, and T. Takahashi. Towards the simulation of dense suspensions: a numerical tool. ESAIM:PROCEEDINGS, 2009. Submitted.

[11] G. Fu and W. Dekelbab. 3-d random packing of polydisperse particles and concrete aggregate grading. Powder Technology, 133:147-155, 2003.

[12] P. Gondret. Hydrodynamique de suspensions monotailles et bitailles en écoulement oscillant. PhD thesis, Université Claude Bernard, 1994

[13] X. Jia, M. Gan, R. A. Williams, and D. Rhodes. Validation of a digital packing algorithm in predicting powder packing densities. Powder Technology, 174:10-13, 2007.

[14] W. S. Jodrey and E. M. Tory. Computer simulation of close packing of equal spheres. Phys. Rev. A, 32:2347-2351, 1985.

[15] A. Lefebvre. Numerical simulation of gluey particles. M2AN, 43:53-80, 2009.

[16] B. D. Lubachevsky, F. H. Stillinger, and E. N. Pinson. Disks vs. spheres: Contrasting properties of random packings. Journal of Statistical Physics, 64:501-524, 1991.

[17] H. A. Makse, D. L. Johnson, and L. M. Schwartz. Packings of compressible granular materials. Phys. Rev. Letters, 84:4160$4163,2000$.

[18] B. Maury. A time-stepping scheme for inelastic collisions. Numerische Mathematik, 102(4):649-679, 2006.

[19] B. Maury and J. Venel. A discrete contact model for crowd motion. Submitted, 2009.

[20] G. E. Mueller. Radial void fraction distributions in randomly packed fixed beds of uniformly sized spheres in cylindrical containers. Powder Technology, 72:269-275, 1992.

[21] G. E. Mueller. Numerically packing spheres in cylinders. Powder Technology, 159:105-110, 2005.

[22] K. Nandakumar, Y. Shu, and K. T. Chuang. Predicting geometrical properties of random packed beds from computer simulation. AIChE Journal, 45:2286-2297, 1999.

[23] G. T. Nolan and P. E. Kavanagh. Random packing of non-spherical particles. Powder Technology, 84:199-205, 1995.

[24] A. P. Philipse. The random contact equation and its implications for (colloidal) rods in packings, suspensions and anisotropic powders. Langmuir, 12(5):1127-1133, 1996.

[25] J.-N. Roux. Geometric origin of mechanical properties of granular materials. Phys. Rev. E, 61:6802, 2000.

[26] S. Sacanna, L. Rossi, A. Wouterse, and A. P. Philipse. Observation of a shape-dependent density maximum in random packings and glasses of colloidal silica ellipsoids. Journal of Physics: Condensed Matter, 19:376108, 2007.

[27] G. D. Scott and D. M. Kilgour. The density of random close packing of spheres. BRIT. J. APPL. PHYS. (J. PHYS. D), 2:863-866, 1969.

[28] S. Siiria and J. Yliruusi. Particle packing simulations based on newtonian mechanics. Powder Technology, 174:82-92, 2007.

[29] L. E. Silbert, D. Ertas, G. S. Grest, T. C. Halsey, and D. Levine. Geometry of frictionless and frictional sphere packings. Phys. Rev. E, 65:031304, 2002.

[30] C. Song, P. Wang, and H. A. Makse. A phase diagram for jammed matter. Nature, 453:629-632, 2008.

[31] S. Torquato, T. M. Truskett, and P. G. Debenedetti. Is random close packing of spheres well defined ? Phys. Rev. Letters, 84:2064-2067, 2000.

[32] S. R. Williams and A. P. Philipse. Random packings of spheres ans spherocylinders simulated by mechanical contraction. Phys. Rev. E, 67:051301, 2003.

[33] A. Wouterse, S. R. Williams, and A. P. Philipse. Effect of particle shape on the density and microstructure of random packing. Journal of Physics: Condensed Matter, 19:406215, 2007.

[34] R. P. Zou and A. B. Yu. Evolution of the packing characteristics of mono-sized non-spherical particles. Powder Technology, 88:71-79, 1996. 